\title{
The encapsulation of an amphiphile into polystyrene microspheres of narrow size distribution
}

\author{
Michal Pellach and Shlomo Margel
}

\begin{abstract}
Encapsulation of compounds into nano- or microsized organic particles of narrow size distribution is of increasing importance in fields of advanced imaging and diagnostic techniques and drug delivery systems. The main technology currently used for encapsulation of molecules within uniform template particles while retaining their size distribution is based on particle swelling methodology, involving penetration of emulsion droplets into the particles. The swelling method, however, is efficient for encapsulation only of hydrophobic compounds within hydrophobic template particles. In order to be encapsulated, the molecules must favor the hydrophobic phase of an organic/aqueous biphasic system, which is not easily achieved for molecules of amphiphilic character. The following work overcomes this difficulty by presenting a new method for encapsulation of amphiphilic molecules within uniform hydrophobic particles. We use hydrogen bonding of acid and base, combined with a pseudo salting out effect, for the entrapment of the amphiphile in the organic phase of a biphasic system. Following the entrapment in the organic phase, we demonstrated, using fluorescein and (antibiotic) tetracycline as model molecules, that the swelling method usually used only for hydrophobes can be expanded and applied to amphiphilic molecules.
\end{abstract}

\section{Findings}

Encapsulation of hydrophobic molecules into hydrophobic particles, on both the nano- and microscale has been applied in drug-delivery systems of lipophilic drugs and in imaging or diagnostic systems [1-6]. Encapsulation allows for protection of the encapsulated material from harsh physical or chemical environments, as well as retention of micro- or nanoparticle surface properties, which may be consequently modified as desired.

Several methods of nano- and microencapsulation have been described in the literature, and the choice of method used depends on hydrophilicity or hydrophobicity of the compound [5]. For hydrophobic compounds, an oil-in-water method is often used, in which the polymer is dissolved together with the compound to be encapsulated in an organic phase. The organic solution is then emulsified in an aqueous continuous phase, and solvent evaporation results in solid particles dispersed in water [5]. Another possibility for entrapment of

* Correspondence: shlomo.margel@mail.biu.ac.il

Department of Chemistry, Bar-Ilan University, Ramat Gan 52900, Israel hydrophobic compounds is by emulsion polymerisation of a hydrophobic vinylic monomer, e.g., styrene, in the presence of the hydrophobic compound. However, these methods present difficulties in obtaining particles of narrow size distribution. The oil-in-water approach as well as a variety of other methods for microencapsulation into biodegradable polylactic acid microparticles has been discussed by Wischke and Schwenderman [6] Modern methods for obtaining monodispersed polymeric microparticles, for example using microporous membrane emulsification [7], have been discussed by VT Tran et al. [8].

Another approach for encapsulation of hydrophobic compounds is a swelling and deswelling method $[4,9]$. The process involves dissolving the compound to be encapsulated in the organic phase, following by emulsifying this organic phase in an appropriate aqueous surfactant solution. When an aqueous suspension of hydrophobic microspheres is introduced into the system, emulsion droplets of organic solution swell the microsphere by slightly separating and entering the sphere between the polymeric chains of which it is composed. 


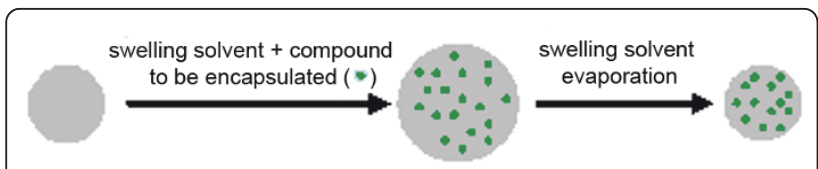

Figure 1 Routine method of encapsulation of a hydrophobic compound into a hydrophobic polymeric microsphere by swelling followed by evaporation of the swelling solvent

The encapsulation is followed by evaporation of the organic swelling solvent, during which the hydrophobic microsphere shrinks back to its near-original size, while the solute remains trapped within the microsphere (Figure 1).

The swelling method of encapsulation may be applied using hydrophobic polymeric microspheres, and is generally limited to encapsulation of hydrophobic substances, e.g. hydrophobic UV absorbing agents [9] or imaging agents $[4,10]$ into polystyrene particles. Amphiphilic substances exhibit solubility problems in organic solvents, and the surfactant in the aqueous phase, used for forming an emulsion, acts as a solubilising agent, increasing the solubility in water and thus further preventing possibility of encapsulation into a hydrophobic matrix. Properties of fluorescein and alkyl derivatives in surfactant micelles have been investigated by Song et al. [11].

Fluorescein has been of great interest to the scientific community due to its interesting chemical properties, its high quantum yield, availability and low costs, as well as its lack of toxicity and approval for in vivo clinical applications [12-14]. Fluorescein is an organic fluorescent molecule with molecular weight $332.3 \mathrm{Da}$, consisting of 3,6-Dihydroxyxanthane, of which the hydroxyl groups give the molecule lipophobic character and two hydrogen bond (H-bond) donors, and phthalolactone, which despite the $\mathrm{H}$-bond acceptors, is largely hydrophobic (structure shown in Figure 2). It is soluble in acetone, slightly soluble in ethanol, but not significantly soluble in other common organic solvents or in (neutral) water [15]. As well as the lipophobic and hydrophobic properties, lack of solubility may be attributed to the pi-stacking interactions between the fluorescein molecules, also held together by $\mathrm{H}$-bonding between hydroxyl groups.

The following work demostrates that fluorescein, an amphiphilic molecule with limited solubility in both water and most common organic solvents, as well as an amphiphilic antibiotic, tetracycline, may be solvated and trapped in the organic phase of a biphasic system, and thus encapsulated into hydrophobic polymeric microspheres. We show that their encapsulation may be performed using methods typically used for hydrophobic molecules and that absolute lipophilicity is not necessarily a prerequisite for encapsulation into a hydrophobic carrier such as polystyrene.

Our findings were that the solubility of fluorescein in dichloromethane (DCM) is increased with addition of triethylamine (TEA). This is probably due to interaction between the nitrogen atom of TEA and the fluorescein hydroxyl groups. In aqueous solutions, fluorescein can exist in a number of $\mathrm{pH}$-dependent forms: cationic, neutral, monoanionic and dianionic (as in Figure 2) $[16,17]$. Presence of TEA thus also increases its solubility in water, with the formation of one or more of the anionic forms of fluorescein, and solute-solvent interactions and $\mathrm{H}$-bonds between the water molecules and hydrophilic groups of the solute molecule $[16,18]$. Solubility in both

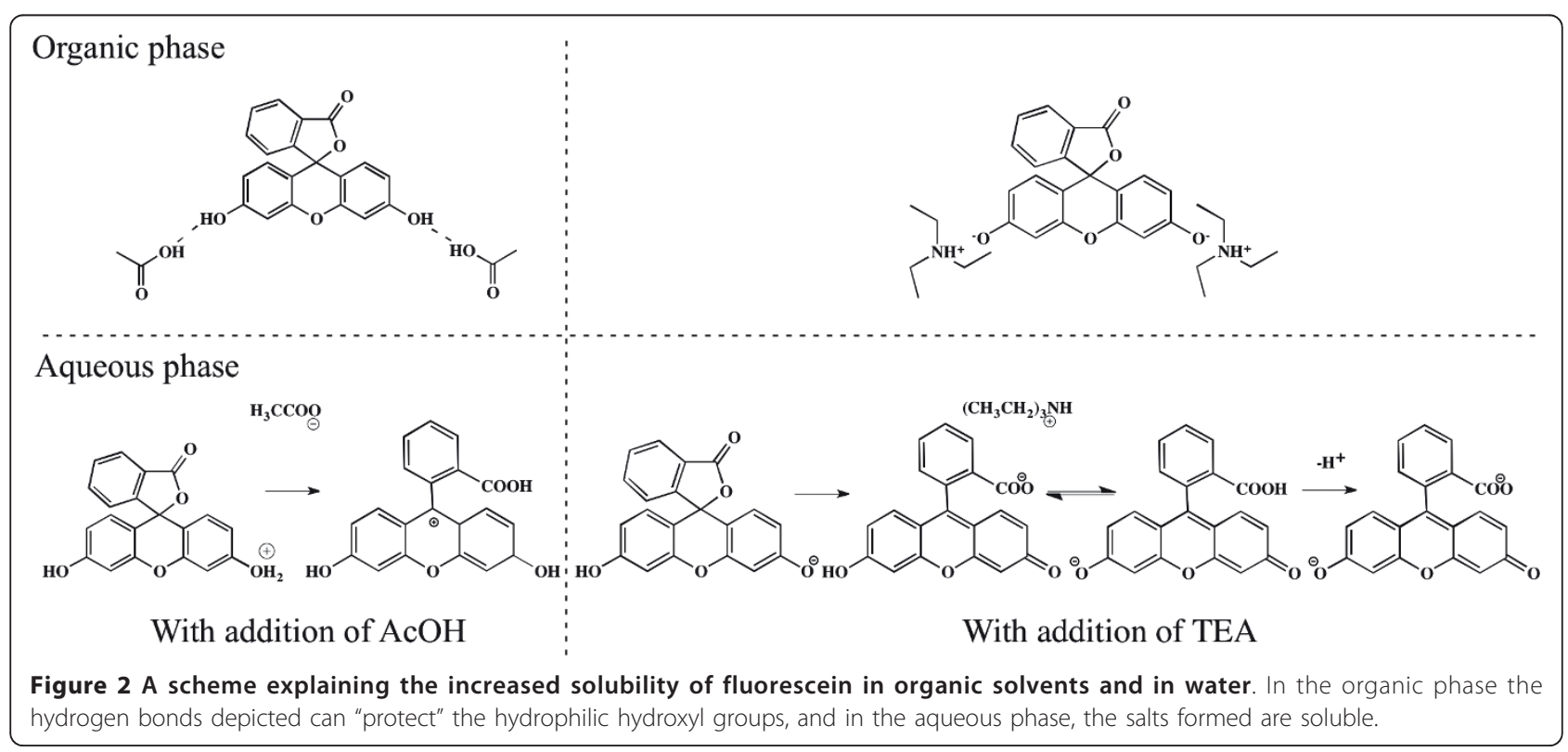


DCM and water is also increased, to a lesser extent, in acidic conditions, with addition of an organic acid such as acetic acid (AcOH). H-bonds may form to sterically protect the hydroxyl groups in an organic solvent, and the formation of the cationic form of fluorescein in the aqueous phase can also occur (Figure 2).

After dissolving fluorescein in DCM containing either TEA or $\mathrm{AcOH}$, on addition of an aqueous solution of the surfactant SDS, as expected, the fluorescein migrates to the aqueous phase, in ionic form. However, when fluorescein was dissolved in DCM, solvated using TEA, followed by addition of $\mathrm{AcOH}$ (a molar equivalent), on addition of an aqueous solution of SDS, the fluorescein remained in the organic phase (Figure 3). The ammonium acetate salt formed appears to have a "salting-out" effect, in which water molecules are attracted to the salt ions and are therefore less available for solvation of the compound in the aqueous phase. This effect in combination with $\mathrm{H}$-bonds formed in the organic phase (e.g. as in Figure 4) together result in entrapment of fluorescein in the organic phase. With less than an equimolar amount of $\mathrm{AcOH}$ added compared to TEA, and therefore less salt formation and migration to the aqueous phase, the fluorescein is distributed throughout both the organic and aqueous phases (Figure 3f). With neutralisation of the basic solution, closure of the lactone ring is allowed, and the increase in ammonium acetate concentration forces the fluorescein back into the organic phase.

Following successful entrapment of fluorescein into the organic phase in a biphasic system, the fluorescein could potentially be encapsulated into polystyrene microspheres by the swelling and deswelling process. Previously synthesised template polystyrene microspheres were used, and their size and size distribution were determined by dynamic light scattering (DLS) with
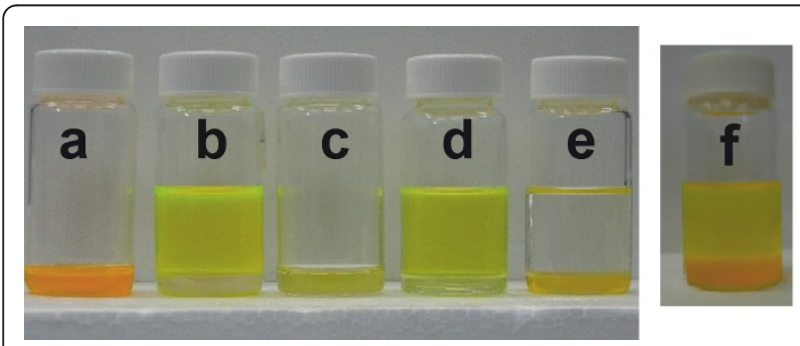

Figure 3 Solvation of fluorescein and its entrapment in the organic phase of a biphasic system: (a) Fluorescein in DCM followed by addition of TEA; (b) Fluorescein in DCM followed by addition of TEA, then aqueous SDS solution; (c) Fluorescein in DCM followed by addition of $\mathrm{ACOH}$; (d) Fluorescein in DCM followed by addition of $\mathrm{AcOH}$, then aqueous SDS solution; (e) Fluorescein in DCM followed by addition of TEA, then a molar equivalent of $\mathrm{ACOH}$, then aqueous SDS solution; (f) same as (e), but with half the quantity of $\mathrm{ACOH}$.

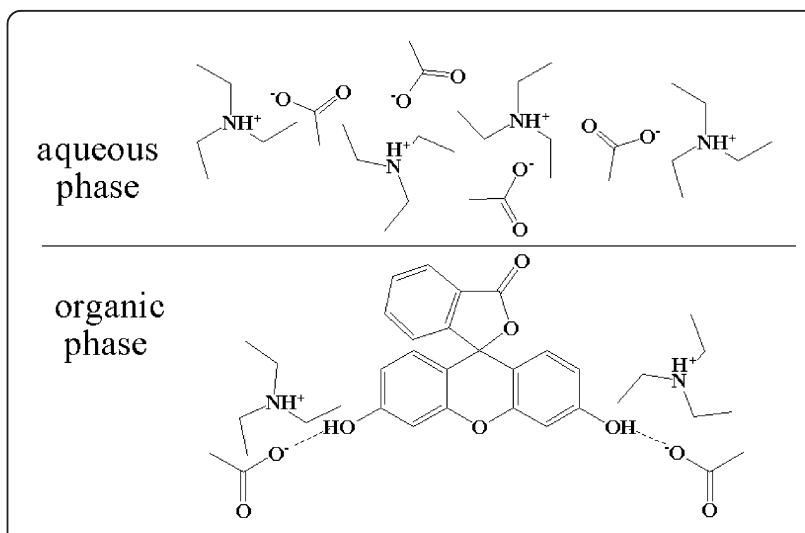

Figure $4 \mathrm{~A}$ scheme explaining the low water solubility of fluorescein in presence of TEA and $\mathrm{AcOH}$.

photon cross-correlation spectroscopy (PCCS) (Figure 5).

Our work illustrated that the optimal time allowed for microsphere swelling while keeping particle uniformity, in a system containing fluorescein, TEA and $\mathrm{AcOH}$, is approximately two hours (Figure 6a). At this stage the size of the template particles has increased from $1.9 \pm$ $0.3 \mu \mathrm{m}$ to $3.2 \pm 0.3 \mu \mathrm{m}$ for the swollen particles. However, the fluorescent particles obtained after evaporation of the swelling solvent, were more or less the same size and size distribution of the orginal polystyrene microspheres. A prolonged amount of time in the swelling conditions, e.g., over $16 \mathrm{~h}$, resulted in non-uniformlysized fluorescent particles, as shown in Figure 6b.

After solvent evaporation, the fluorescein-containing microspheres (Figure 7) were washed with water, removing the SDS and excess ammonium acetate. The image obtained by confocal microscopy (Figure $8 \mathrm{~A}$ ), representing a slice through the centre of the beads, showed that

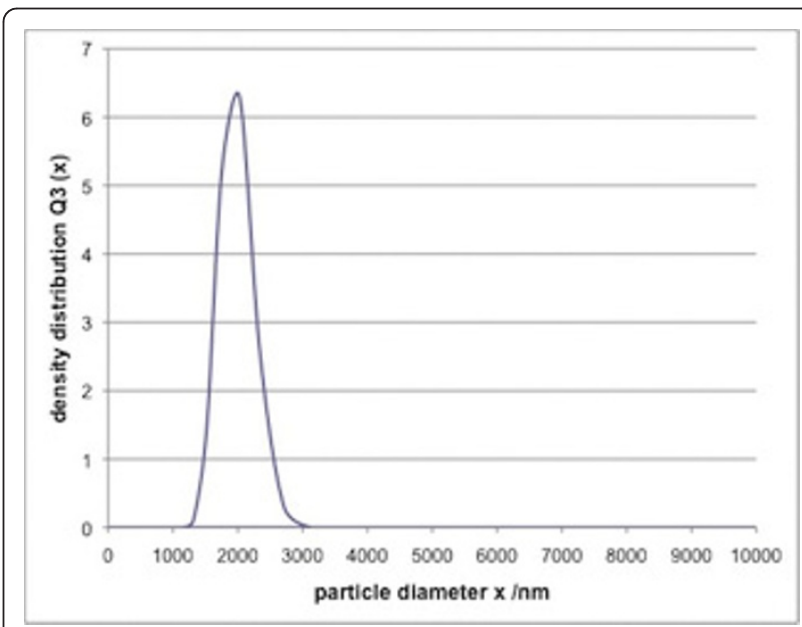

Figure 5 Size and size distribution of template polystyrene nanoparticles, determined by DLS with PCCS. 


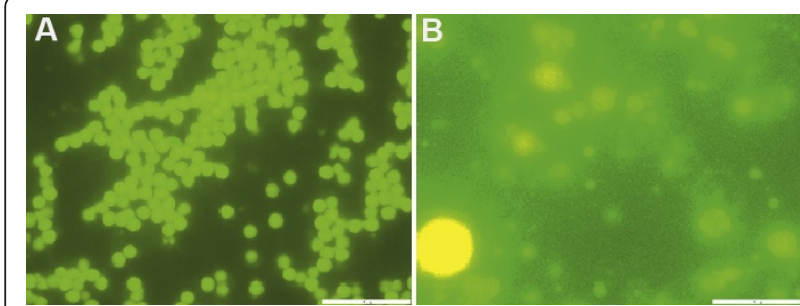

Figure 6 Fluorescent microscopy of swollen microspheres after $2 \mathrm{~h}$ (a) and after $16 \mathrm{~h}$ (b) swelling with fluorescein dissolved in DCM. Scale bar $=20 \mu \mathrm{m}$.

fluorescein was distributed throughout the microsphere and not merely adsorbed on the surface, as shown for comparison in Figure 8B. There was no significant modification of the fluorescein molecule, as shown by the fluorescent microscopy and the fluorescence spectra, the beads having similar fluorescence properties to fluorescein in solution. (Figures 7, 8 and 9).

In order to show that our model was not solely applicable to fluorescein, we searched for a molecule with similar properties. Tetracycline is a widely available antibiotic, with amphiphilic properties. Its planar ring structure gives it hydrophobic properties, and its primary amine and five hydroxyl groups give it hydrophilic character (Figure 10A). Tetracycline is somewhat soluble in DCM, and while its solubility in certain organic solvents may be considered adequate, on addition of an aqueous surfactant solution tetracycline behaves similarly to fluorescein and migrates to the aqueous phase. Simple salting out, using brine, did not achieve remigration back to the organic phase. However, treatment of a solution of tetracycline in DCM with TEA followed by a molar equivalent of $\mathrm{AcOH}$ resulted in successful

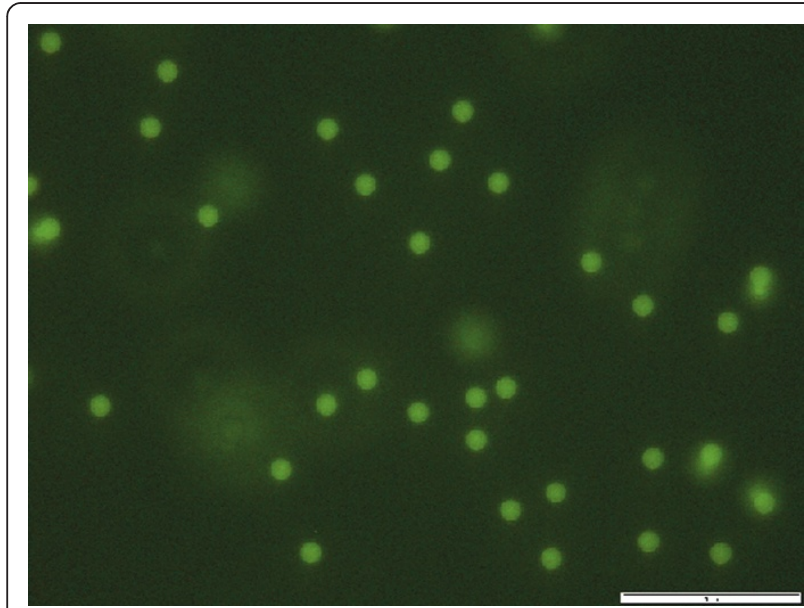

Figure 7 Fluorescent microscope image of washed $1.9 \pm 0.3$ $\mu \mathrm{m}$ fluorescein-containing polystyrene microspheres. Scale bar $=20 \mu \mathrm{m}$.

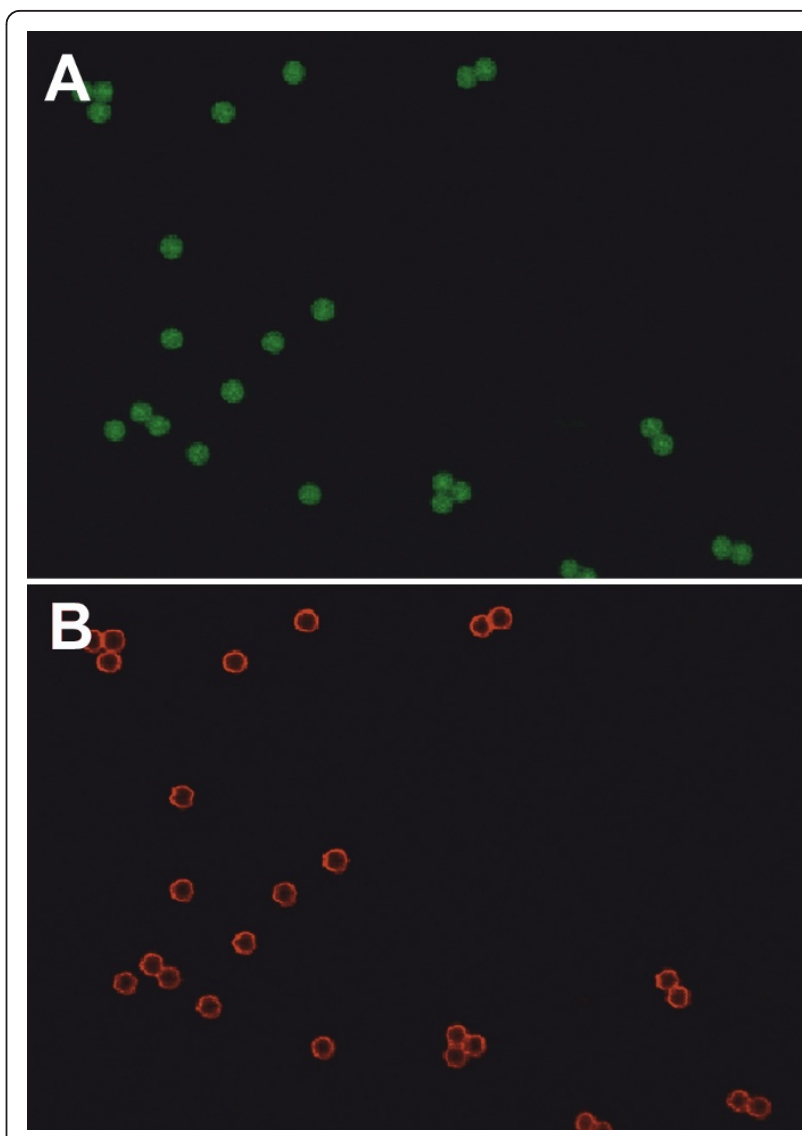

Figure 8 Confocal microscopy, representing a slice through the centre of the bead: (A) shows that the fluorescein is distributed throughout the microsphere and not simply adsorbed on the bead surface, while (B) shows rhodamine on the surface of polystyrene microspheres, displayed solely for comparison, appearing as rings around the beads. These particles were coated with gelatine, and rhodamine isothiocyanate was bound to the gelatine amines. Original Magnification $=\times 600$.

entrapment in the organic phase, also in presence of an SDS aqueous solution (Figure 10B). Following entrapment, tetracycline could also be encapsulated into polystyrene, using swelling and deswelling. Since the fluorescence quantum yield of tetracycline is low $[19,20]$, the resulting fluorescent signal of the polystyrene microspheres containing the tetracycline is relatively week, as shown in Figure 10C.

Overall, our results show the entrapment of amphiphilic molecules into a hydrophobic environment, achieved by solvation, and by controlling the molecular surroundings. Until now, encapsulation using the method of swelling, which allows for retention of uniform particle size distribution, has been efficient only for encapsulation of hydrophobic substances into hydrophobic particles. The present work suggests a solution to problems with reagent solubility, and expands the swelling method to also include amphiphilic substances. 


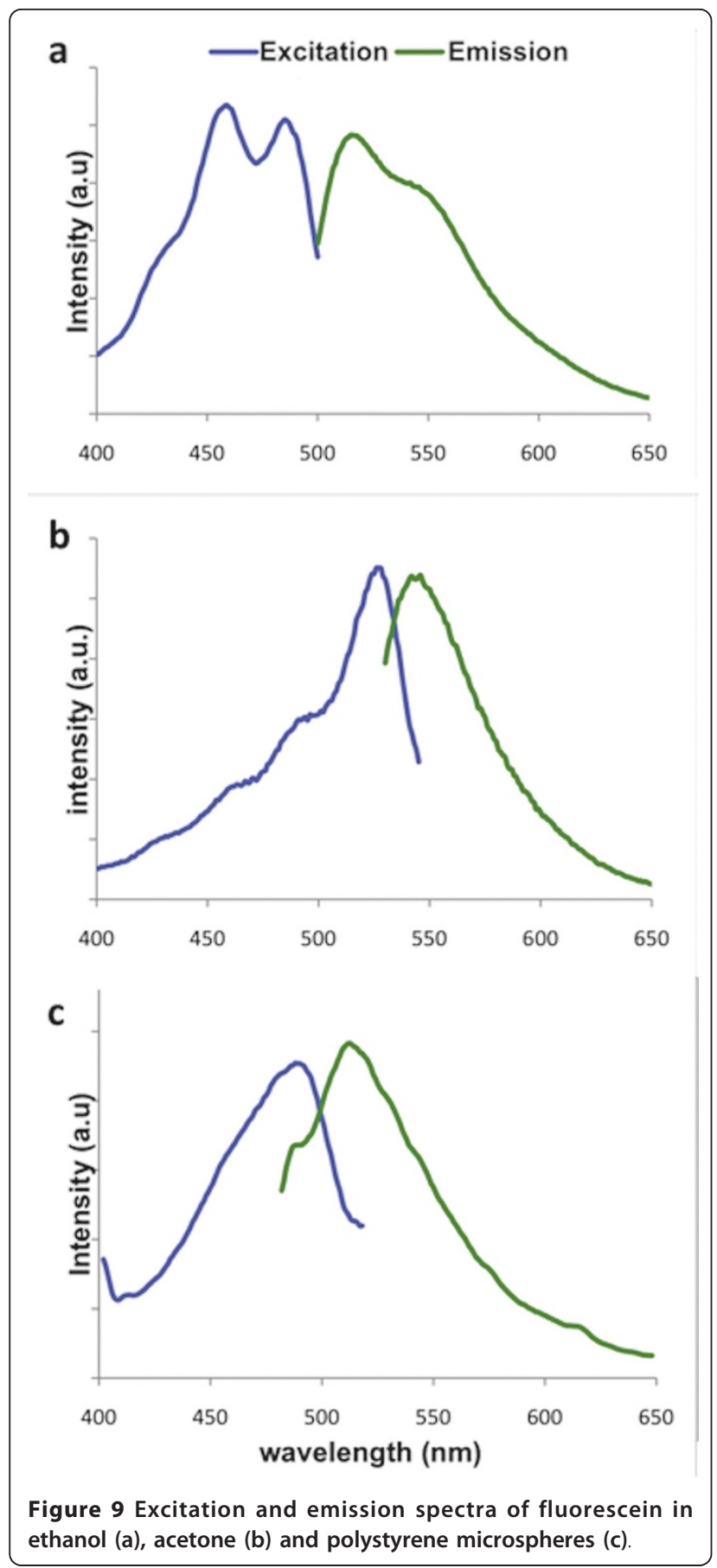

In future, we plan to extend this work for additional amphiphiles and hydrophobic polymeric particles, e.g., biodegradable polymers such as polymethylmethacrylate and polylactic acid. Future plans also include coating of the hydrophobic polymeric particles with functional groups, so that they may be conjugated to a targeting agent, and used for purposes of diagnostics or targeted drug delivery.
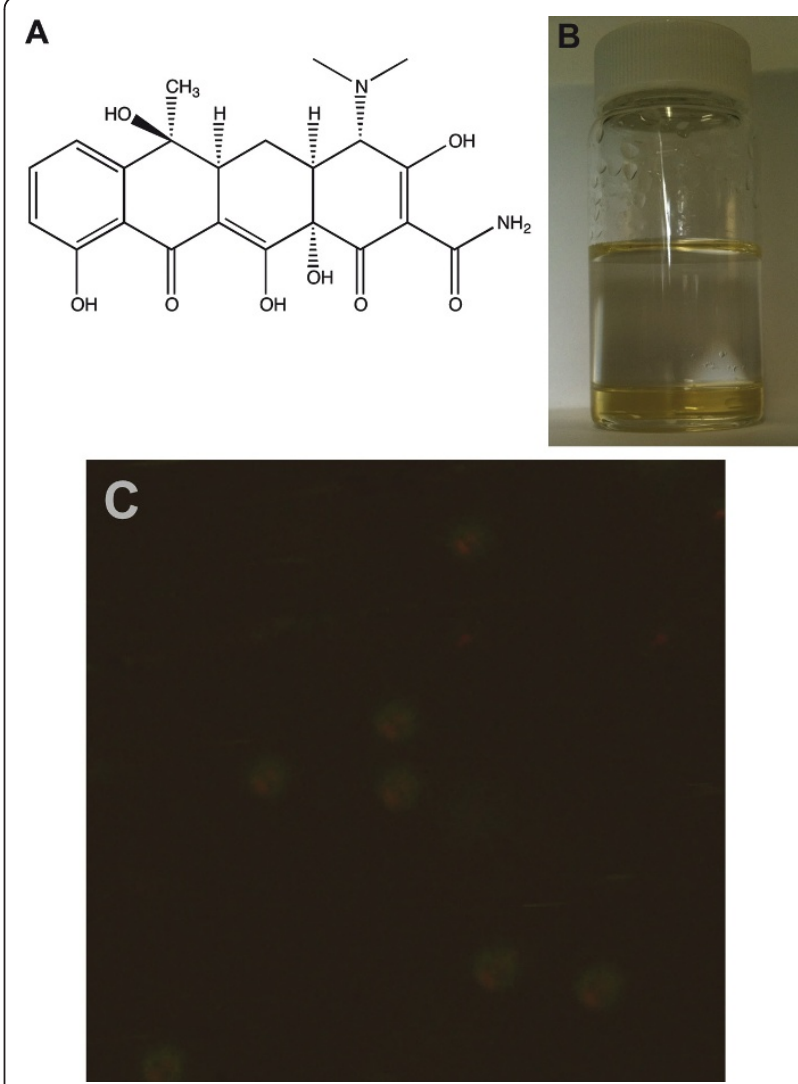

Figure 10 Tetracycline $(A)$, its entrapment into the organic phase of a biphasic system (B) and its encapsulation into polystyrene microspheres $(C)$ Original magnification $\times 600$.

\section{Experimental}

Synthesis of polystyrene microspheres

Polystyrene microspheres of $1.9 \pm 0.3 \mu \mathrm{m}$ were synthesised in our lab by dispersion polymerisation, as previously described $[4,21]$. Briefly, polyvinylpyrrolidone (MW $360000,1.5 \% \mathrm{w} / \mathrm{v}$ of total solution), dissolved in a mixture of ethanol $(150 \mathrm{~mL})$ and 2 -methoxyethanol $(62.5 \mathrm{~mL})$, was introduced into a $1 \mathrm{~L}$ flask. The solution was deaerated using nitrogen, heated to $73^{\circ} \mathrm{C}$, and a deaerated solution of benzoyl peroxide $(1.5 \mathrm{~g})$ in styrene $(37.5 \mathrm{~mL})$ was then added. The polymerisation continued under nitrogen for $24 \mathrm{~h}$, and was terminated by cooling. The microspheres formed were washed by centrifugation with ethanol followed by water, and then dried by lyophilisation. The diameter of the microspheres is variable, and was controlled by varying parameters such as monomer, initiator or stabiliser concentrations.

\section{Swelling and deswelling method of encapsulation}

TEA $(70 \mu \mathrm{L}, 0.5 \mathrm{mmol})$ was added to fluorescein $(0.4$ $\mathrm{mg}, 1.2 \mu \mathrm{mol})$ or tetracycline $(2 \mathrm{mg}, 4.5 \mu \mathrm{mol})$ in DCM 
$(2 \mathrm{~mL})$, followed by addition of $\mathrm{AcOH}(30 \mu \mathrm{L}, 0.5$ $\mathrm{mmol}$ ). An (immiscible) aqueous solution of $1 \%$ sodium dodecyl sulphate (SDS, $10 \mathrm{~mL}$ ) was added to the organic solution. The mixture was then sonicated for one minute to give emulsion droplets containing the fluorescein or tetracycline. An aqueous 7\% suspension of template polystyrene microspheres $(3.5 \mathrm{~mL}, 245 \mathrm{mg}$ microspheres) was then added to the emulsion, and the mixture was allowed to stir. The swelling process is complete when all the droplets have diffused into the microspheresas verified by light microscopy. The disappearance of the emulsion droplets containing the amphiphile from the aqueous phase is also indication for the complete entrapment of the fluorescein molecules within the polystyrene template microspheres. The swelling solvent was then slowly evaporated, at room temperature. The microspheres were then washed with water by repeated centrifugation cycles.

\section{Particle Size and size distribution}

Particle size and size distribution was determined by DLS with PCCS (Nanophox particle analyser, Sympatec $\mathrm{GmbH}$, Germany).

\section{Microscopy}

Fluorescent micrographs were obtained with an Olympus microscope, model BX51, or Zeiss microscope, model LSM510. Confocal microscopy was employed for verification of encapsulation of fluorescent material, and was performed using an Olympus FV1000 confocal microscope.

\section{Authors' contributions}

MP carried out the work described in the paper, under supervision of SM. MP and SM read and approved the final manuscript.

\section{Competing interests}

The authors declare that they have no competing interests.

Received: 31 August 2011 Accepted: 6 December 2011

Published: 6 December 2011

\section{References}

1. Torchilin VP: Micellar Nanocarriers: Pharmaceutical Perspectives. Pharmaceutical Research 2007, 24:1-16.

2. Gaucher G, Dufresne M-H, Sant VP, Kang N, Maysinger D, Leroux J-C: Block copolymer micelles: preparation, characterization and application in drug delivery. Journal of Controlled Release 2005, 109:169-188.

3. Doiron AL, Homan KA, Emelianov S, Brannon-Peppas L: Poly(Lactic-coGlycolic) Acid as a Carrier for Imaging Contrast Agents. Pharmaceutical Research 2009, 26:674-682.

4. Galperin A, Margel D, Margel S: Synthesis and characterization of uniform radiopaque polystyrene microspheres for X-ray imaging by a single-step swelling process. Journal of Biomedical Materials Research Part A 2006, 79A:544-551.

5. Li M, Rouaud O, Poncelet D: Microencapsulation by solvent evaporation: State of the art for process engineering approaches. International Journal of pharmaceutics 2008, 363:26-39.
6. Wischke C, Schwendeman SP: Principles of encapsulating hydrophobic drugs in PLA/PLGA microparticles. International Journal of pharmaceutics 2008, 364:298-327.

7. Meyer RF, Rogers WB, McClendon MT, Crocker JC: Producing Monodisperse Drug-Loaded Polymer Microspheres via Cross-Flow Membrane Emulsification: The Effects of Polymers and Surfactants. Langmuir 2010, 26:14479-14487.

8. Tran V-T, Benoit J-P, Venier-Julienne M-C: Why and how to prepare biodegradable, monodispersed, polymeric microparticles in the field of pharmacy? International Journal of Pharmaceutics 2011, 407:1-11.

9. Goldshtein J, Margel S: Synthesis and characterization of polystyrene/2(5-chloro-2H-benzotriazole-2-yl)-6-(1,1- dimethylethyl)-4-methyl-phenol composite microspheres of narrow size distribution for UV irradiation protection. Colloid \& Polymer Science 2011, 1-12.

10. Behnke T, Wurth C, Hoffmann K, Hubner M, Panne U, Resch-Genger U: Encapsulation of Hydrophobic Dyes in Polystyrene Micro- and Nanoparticles via Swelling Procedures. Journal of Fluorescence 2011, 21:937-944.

11. Song A, Zhang J, Zhang M, Shen T, Tang J: Spectral properties and structure of fluorescein and its alkyl derivatives in micelles. Colloids and Surfaces A: Physicochemical and Engineering Aspects 2000, 167:253-262.

12. Kapadia GJ, Tokuda H, Sridhar R, Balasubramanian V, Takayasu J, Bu P, Enjo F, Takasaki M, Konoshima T, Nishino H: Cancer chemopreventive activity of synthetic colorants used in foods, pharmaceuticals and cosmetic preparations. Cancer Letters 1998, 129:87-95.

13. Burchak ON, Mugherli L, Chatelain F, Balakirev MY: Fluorescein-based amino acids for solid phase synthesis of fluorogenic protease substrates. Bioorganic \& Medicinal Chemistry 2006, 14:2559-2568.

14. Du X, Zhang H, Guo X, Deng Y, Wang H: 6-Oxy-(acetyl piperazine) fluorescein as a new fluorescent labeling reagent for free fatty acids in serum using high-performance liquid chromatography. Journal of Chromatography A 2007, 1169:77-85.

15. Green FJ: The Sigma-Aldrich Handbook of Stains, Dyes and Indicators Milwaukee, Wisconsin: Aldrich Chemical Company; 1990.

16. Krol M, Wrona M, Page CS, Bates PA: Macroscopic pKa Calculations for Fluorescein and Its Derivatives. Journal of Chemical Theory and Computation 2006, 2:1520-1529.

17. Klonis N, Sawyer WH: Spectral properties of the prototropic forms of fluorescein in aqueous solution. Journal of Fluorescence 1996, 6:147-157.

18. Almerindo Gl, Tondo DW, Pliego JR: lonization of Organic Acids in Dimethyl Sulfoxide Solution: A Theoretical Ab Initio Calculation of the pKa Using a New Parametrization of the Polarizable Continuum Model. The Journal of Physical Chemistry A 2003, 108:166-171.

19. Kaszycki P, Guz A, Drwięga M, Wasylewski Z: Tet repressor-tetracycline interaction. Journal of Protein Chemistry 1996, 15:607-619.

20. Popov PG, Vaptzarova KI, Kossekova GP, Nikolov TK: Fluorometric study of tetracycline-bovine serum albumin interaction: The tetracyclines-A new class of fluorescent probes. Biochemical Pharmacology 1972, 21:2363-2372.

21. Bamnolker H, Margel S: Dispersion Polymerization of Styrene in Polar Solvents: Effect of Reaction Parameters on Microsphere Surface Composition and Surface Properties, Size and Size Distribution, and Molecular Weight. Journal of Polymer Science: Part A: Polymer Chemistry 1996, 34:1857-1871.

doi:10.1186/1752-153X-5-78

Cite this article as: Pellach and Margel: The encapsulation of an amphiphile into polystyrene microspheres of narrow size distribution. Chemistry Central Journal 2011 5:78. 